\title{
Commentary: How to predict disaster?
}

\author{
Hiroshi Date, MD
}

\author{
From the Department of Thoracic Surgery, Graduate School of Medicine, Kyoto University, Kyoto, Japan. \\ Disclosures: Author has nothing to disclose with regard to commercial support. \\ Received for publication Aug 14, 2019; revisions received Aug 14, 2019; accepted for publication Aug 19, 2019; \\ available ahead of print Oct 13, 2019. \\ Address for reprints: Hiroshi Date, MD, Department of Thoracic Surgery, Graduate School of Medicine, Kyoto \\ University, 54 Kawahara-cho, Shogoin, Sakyo-ku, Kyoto 606-8507, Japan (E-mail: hdate@kuhp.kyoto-u.ac. \\ jp). \\ J Thorac Cardiovasc Surg 2020;159:1119 \\ $0022-5223 / \$ 36.00$ \\ Copyright (c) 2019 by The American Association for Thoracic Surgery \\ https://doi.org/10.1016/j.jtcvs.2019.08.124
}

It is well known that idiopathic pulmonary fibrosis (IPF) is associated with an increased risk of lung cancer. Surgical treatment for patients with lung cancer with IPF may provoke acute exacerbation (AE) of IPF. Clinical features of $\mathrm{AE}$ include worsening or development of dyspnea, new radiologic infiltration, and poor oxygenation. Once $\mathrm{AE}$ occurs, it is associated with mortality rates between $33.3 \%$ and $100 \%$. It is indeed a disastrous postoperative complication for which no effective treatment has been established. According to the annual report by The Japanese Association for Thoracic Surgery, ${ }^{1}$ hospital mortality rate after lung cancer surgery was $0.6 \%$ and $\mathrm{AE}$ was the leading cause of death.

To avoid this disastrous complication, it is very important to identify risk factors of AE. In our previous report on 1763 patients with lung cancer with interstitial lung diseases including 1235 patients with IPF, we identified following 7 independent risk factors of AE: surgical procedures, male sex, history of exacerbation, preoperative steroid use, serum sialylated carbohydrate antigen KL-6 levels, usual interstitial pneumonia appearance on computed tomography scan, and reduced percent predicted vital capacity. ${ }^{2}$ Among these, surgical procedures showed the strongest association with $\mathrm{AE}$ (using wedge resection as the reference, lobectomy or segmentectomy: odds ratio, 3.83 ; $95 \%$ confidence interval, 1.94-7.57; bi-lobectomy or pneumonectomy: odds ratio, 5.70; 95\% confidence interval, 2.38-13.7; $P<.001)$.

In this issue of the Journal, Yamamichi and colleagues ${ }^{3}$ demonstrate that the maximum standardized uptake values of non-lung tumor area (NTL-SUVmax) on fluorodeoxyglucose-positron emission tomography (PET) may be used to predict postoperative AE after surgical resection for patients with lung cancer associated with IPF. They identified 822 patients including 120 patients with IPF. Among 822 patients, AE occurred in 15 patients

\section{References}

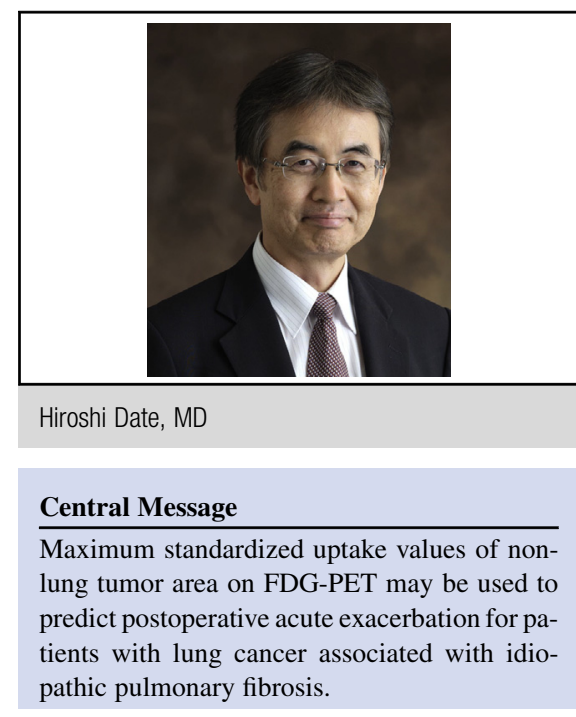

See Article page 1111.

$(1.8 \%)$. Among 120 patients with IPF, AE occurred in 12 patients $(10 \%)$. With multivariate analysis, the NTL-SUVmax was identified as an independent predictive factor for AE. It is known that fluorodeoxyglucosePET can measure cellular glucose metabolism and PET signals an increase in the area of active inflammation. ${ }^{4}$ The idea of evaluating NTL-SUVmax is unique and interesting, because it may be reflecting the activity of inflammatory process in patients with IPF.

It would be interesting if NTL-SUVmax is correlated with our previously reported 7 risk factors of $\mathrm{AE}$, a disastrous complication.

1. Sato T, Teramukai S, Kondo H, Watanabe A, Ebina M, Kishi K, et al. Impact and predictors of acute exacerbation of interstitial lung diseases after pulmonary resection for lung cancer. J Thorac Cardiovasc Surg. 2014;147:1604-11.

2. Committee for Scientific Affairs, The Japanese Association for Thoracic Surgery, Shimizu H, Endo S, Natsugoe S, Doki Y, et al. Thoracic and cardiovascular surgery in Japan in 2016: annual report by the Japanese Association for Thoracic Surgery. Gen Thorac Cardiovasc Surg. 2019;67:377-411.

3. Yamamichi T, Shimada Y, Masuno R, Ohira T, Abe S, Yoshimura A, et al. Association between F-18 fluorodeoxyglucose uptake of noncancerous lung area and acute exacerbation of interstitial pneumonia in patients with lung cancer after resection. J Thorac Cardiovasc Surg. 2020;159:1111-8.e2.

4. Groves AM, Win T, Screaton NJ, Berovic M, Endozo R, Booth H, et al. Idiopathic pulmonary fibrosis and diffuse parenchymal lung disease: implications from initial experience with 18F-FDG PET/CT. J Nucl Med. 2009;50:538-45. 